\title{
Fluctuations of Goldstone modes and the chiral transition in QCD
}

\author{
Frithjof Karsch (RBC-Bielefeld collaboration) \\ Physics Department, Brookhaven National Laboratory, Upton, NY 1973, USA \\ and Fakultät für Physik, Universität Bielefeld, D-33615 Bielefeld, Germany
}

\begin{abstract}
We provide evidence for the influence of thermal fluctuations of Goldstone modes on the chiral condensate at finite temperature. We show that at fixed temperature, $T<T_{c}$, in the vicinity of the chiral transition temperature this leads to a characteristic dependence of the chiral condensate on the square root of the light quark mass $\left(m_{l}\right)$, which is expected for 3-dimensional models with broken $\mathrm{O}(\mathrm{N})$ symmetry. As a consequence the chiral susceptibility shows a strong quark mass dependence for all temperatures below $T_{c}$ and diverges like $1 / \sqrt{m_{l}}$ in the chiral limit.
\end{abstract}

Key words: QCD thermodynamics, chiral symmetry breaking, Goldstone modes PACS: 11.15.Ha, 11.10.Wx, 12.38.Gc, 12.38.Mh

\section{Introduction}

Establishing the properties of the chiral phase transition in QCD is one of the outstanding problems in lattice simulations of QCD thermodynamics. Although several attempts have been undertaken to establish the unique scaling behavior in the limit of vanishing light quark masses no clear-cut evidence for the expected $O(N)$ scaling, related to the underlying chiral symmetry of the QCD Lagrangian, has been found. This may have several reasons related to common problems in lattice calculations: cut-off effects, finite volume effects, explicit flavor symmetry breaking and/or too large quark masses.

We will discuss here yet another effect that influences the scaling behavior of thermodynamic quantities in the vicinity of the finite temperature chiral transition of QCD and may compete with the universal scaling in the vicinity of $T_{c}$. This is related to singularities in chiral observables, e.g. the chiral susceptibility, that are induced at ANY

Email address: karsch@bnl.gov (Frithjof Karsch (RBC-Bielefeld collaboration)). 

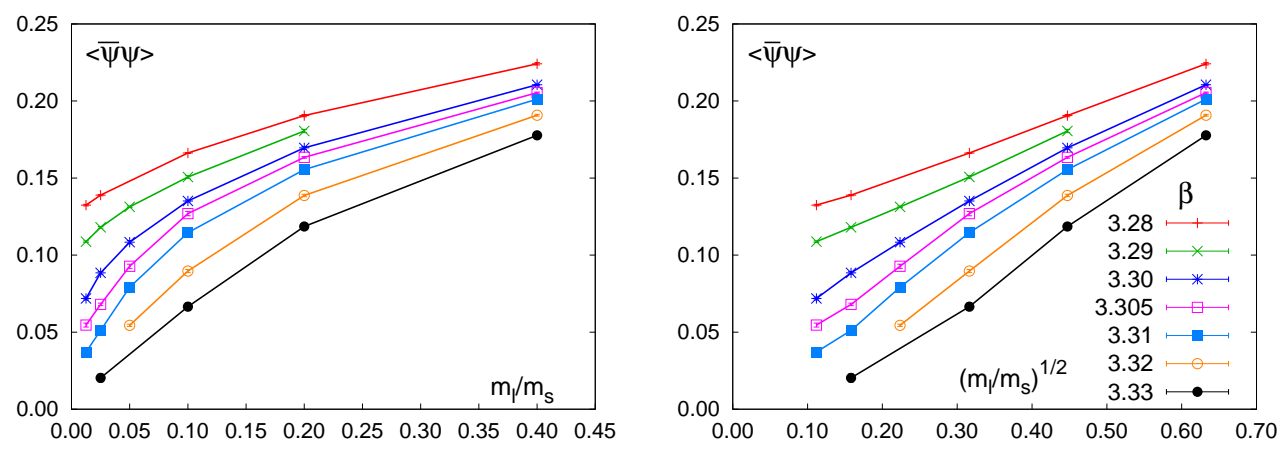

Fig. 1. Chiral condensate calculated on lattices of size $N_{\sigma}^{3} \times 4$ in $(2+1)$-flavor QCD for various values of the light quark mass $\left(m_{l}\right)$ in units of the strange quark mass $\left(m_{s}\right) . N_{\sigma}$ from 32 for the two lightest quark mass values to 8 for the heaviest masses. For small quark masses the critical coupling for the chiral transition temperature on this size lattices has been determined to be in the range $\beta_{c}=3.305-3.31$ [ .

temperature in the chiral symmetry broken phase. The existence of these singularities induced by fluctuations of Goldstone modes are well known from the analysis of 3 and 4 dimensional statistical models and field theories with global $O(N)$ symmetries that are spontaneously broken at low temperature $[12314]$.

\section{Chiral condensate and susceptibility for $T \lesssim T_{c}$}

At vanishing temperature properties of $\mathrm{QCD}$ in the light quark sector are described by an effective 4-d, $O(4)$ symmetric Lagrangian; chiral symmetry is broken and, what is of interest here, at non-zero quark mass the light quark chiral condensate, $\langle\bar{\psi} \psi\rangle$, receives logarithmic corrections proportional to $m_{l} \ln m_{l}$ that are due to the presence of light Goldstone modes 2]. This leads to a logarithmic divergence of the chiral susceptibility, $\chi_{m} \sim \mathrm{d}\langle\bar{\psi} \psi\rangle / \mathrm{d} m$. In three dimensions the analog to these logarithmic terms are corrections that are proportional to the square root of the quark mass [1. As a consequence $\chi_{m}$ will diverge in the chiral limit like $\chi_{m} \sim 1 / \sqrt{m_{l}}$.

In the vicinity of the QCD phase transition a $3-\mathrm{d}, O(4)$ symmetric effective theory is expected to describe the universal properties of the transition [5] which will also control the properties of the chiral condensate for temperatures close to $T_{c}$. We thus expect that in the chiral limit $\chi_{m}$ will diverge for all values of the temperature $T \leq T_{c}$,

$$
\chi_{m} \sim \begin{cases}m_{l}^{-1 / 2} & , T \leq T_{c} \\ m_{l}^{1 / \delta-1} & , T=T_{c}\end{cases}
$$

where $1 / \delta-1 \simeq 0.79$ for 3 -d $O(4)$ as well as $O(2)$ symmetric spin models.

The appearance of a square root singularity has, in fact, been established in the vicinity of the chiral phase transition of an $S U(3)$ gauge theory coupled to adjoint fermions [6] where it is apparent already for moderately small values of the quark mass. Similar evidence for the role of light Goldstone modes in QCD, however, has been missing so far.

In Fig. 1 we show the light quark chiral condensate, $\langle\bar{\psi} \psi\rangle$ calculated in $(2+1)$-flavor QCD with improved staggered fermions (p4-action) on lattices of size $N_{\sigma}^{3} \times 4$ for various values of the gauge coupling. The figures show results for $\langle\bar{\psi} \psi\rangle$, plotted versus the light 

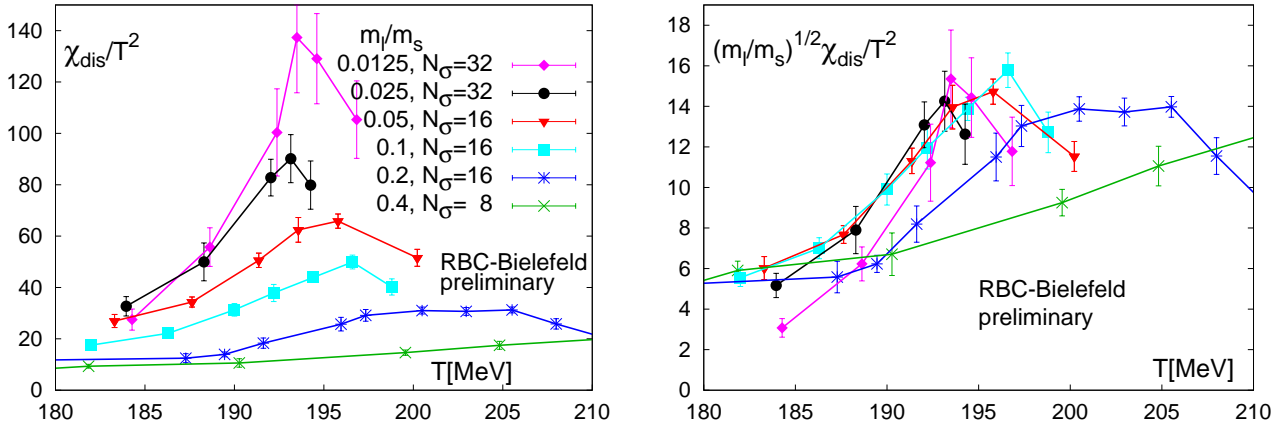

Fig. 2. Disconnected part of the chiral susceptibility calculated on lattices of size $N_{\sigma}^{3} \times 4$ in (2+1)-flavor QCD for various values of the light quark mass.

quark mass $\left(m_{l}\right)$ expressed in units of the strange quark mass $\left(m_{s}\right)$ (left) and the square root of this ratio (right). The right hand figure indeed suggests that the strong curvature of $\langle\bar{\psi} \psi\rangle$ as function of $m_{l}$ is well accounted for by a $\sqrt{m_{l}}$ dependence for $T<T_{c}$. In any case, the weaker than linear dependence of $\langle\bar{\psi} \psi\rangle$ on $m_{l}$ leads to a strong quark mass dependence of the chiral susceptibility, $\chi_{m}$. This susceptibility receives two contributions, usually referred to as disconnected and connected parts, $\chi_{m}=\chi_{\text {dis }}+\chi_{c o n}$, with

$$
\frac{\chi_{\text {dis }}}{T^{2}}=\frac{N_{\tau}}{16 N_{\sigma}^{3}}\left(\left\langle\left(\operatorname{Tr} D_{l}^{-1}\right)^{2}\right\rangle-\left\langle\operatorname{Tr} D_{l}^{-1}\right\rangle\right), \frac{\chi_{c o n}}{T^{2}}=-\frac{N_{\tau}^{2}}{4} \sum_{x}\left\langle D_{l}^{-1}(x, 0) D_{l}^{-1}(0, x)\right\rangle .
$$

Here $D_{l}$ denotes the staggered fermion matrix for light quarks. In general, both contributions are sensitive to the Goldstone modes and will receive divergent contributions, $\chi_{d i s, c o n} \sim 1 / \sqrt{m_{l}}$ 4. We will restrict our analysis here to the disconnected part.

In Fig. 2 we show $\chi_{\text {dis }} / T^{2}$ calculated for various values of $m_{l}$. In these calculations the strange quark mass is close to its physical value and $m_{l}$ reaches values well below the physical one; a ratio $m_{l} / m_{s} \simeq 0.04$ corresponds in the continuum limit to the physical quark mass ratio. The strong quark mass dependence in a wide temperature interval is obvious from Fig. 2(left); its dominant contribution in the low temperature regime is indeed well described by $\chi_{m} \sim 1 / \sqrt{m_{l}}$ as can be seen from the rescaled data shown in Fig. 2(right). We note that this seems to hold for all quark masses, $m_{l} \leq 0.1 m_{s}$, although at present our data sample for the lightest quark mass is not yet good enough to substantiate this statement also for this light mass value, which corresponds to a light pseudo-scalar mass of about $70 \mathrm{MeV}$. We furthermore, note that the square root behavior also dominates the quark mass dependence in the peak region, i.e. there is no evidence for a stronger singularity, which is expected to arise from the singular part of the partition function of 3-d, $\mathrm{O}(4)$ (or $\mathrm{O}(2)$ ) symmetric models, $\chi_{m}^{\text {singular }} \sim m_{l}^{-0.79}$.

\section{Towards the continuum limit of $(2+1)$-flavor QCD}

The general features of the quark mass dependence of the disconnected part of the chiral susceptibility are also apparent in calculations currently performed by the hotQCD collaboration on lattice with temporal extent $N_{\tau}=8[8$, i.e. closer to the continuum limit. In Fig. 3 we show $\chi_{d i s} / T^{2}$ and the rescaled susceptibility. The left hand part of the 

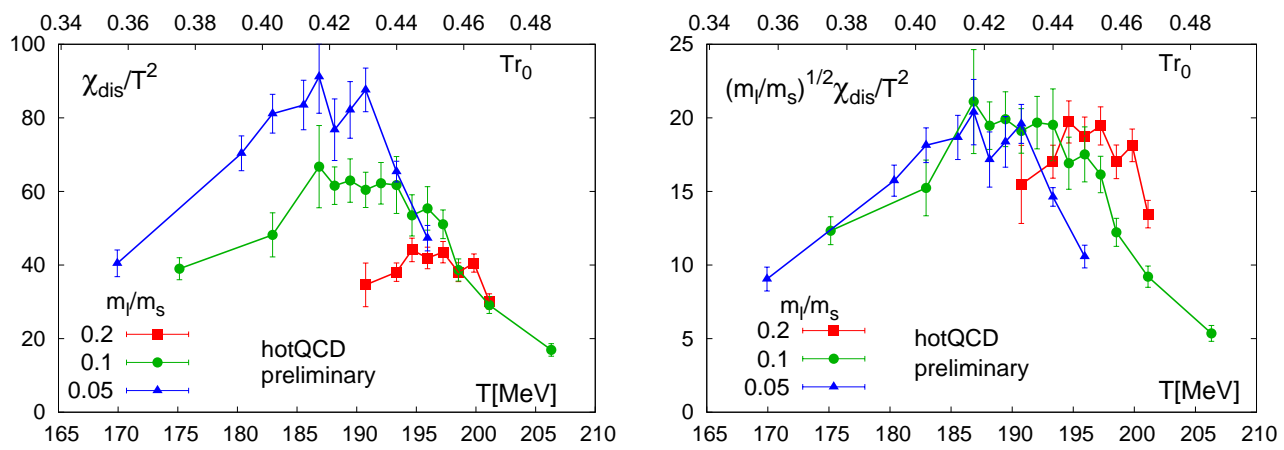

Fig. 3. Disconnected part of the chiral susceptibility calculated on lattices of size $32^{3} \times 8$ in $(2+1)$-flavor QCD for $m_{l} / m_{s}=0.05,0.1$ and 0.2 , respectively.

figure shows that for temperatures above the transition region the susceptibilities quickly become quark mass independent. This is expected as the chiral condensates will be linear in the quark mass in the chirally symmetric phase of QCD. Below the transition region we again observe a strong quark mass dependence that is consistent with $\sim 1 / \sqrt{m_{l}}$.

The weak quark mass dependence of $\chi_{m}$ in the high temperature regime suggest that the susceptibility can already be described by the limiting form for the $m_{l} \rightarrow 0$ limit,

$$
\chi_{m}= \begin{cases}\infty & , T \leq T_{c} \\ A\left(\frac{T-T_{c}}{T_{c}}\right)^{-\gamma} & , T>T_{c}\end{cases}
$$

A consistent picture for the chiral susceptibility thus seems to emerge despite the missing evidence for $O(N)$ scaling for its peak height.

Acknowledgments The numerical calculations presented here have been carried out on the QCDOC supercomputers of the RIKEN-BNL Research Center, the BlueGene/L computers at the New York Center for Computational Sciences and the LLNL. The work of FK has been supported by DOE under Contract No. DE-AC02-98CH10886.

\section{References}

[1] D.J.Wallace and R.K.P. Zia, Phys. Rev. B12 (1975) 5340

[2] J. Gasser and H. Leutwyler, Phys. Lett. B184 (1987) 83

[3] P. Hasenfratz and H. Leutwyler, Nucl. Phys. B343 (1990) 241

[4] A. V. Smilga, Phys. Lett. B318 (1993) 531;

A. V. Smilga and J. J. M. Verbaarschot, Phys. Rev. D54 (1996) 1087

[5] R. Pisarski and F. Wilczek, Phys. Rev. D29 (1984) 338

[6] F. Karsch and M. Lütgemeier, Nucl. Phys. B550 (1999) 449;

J. Engels, S. Holtmann and T. Schulze, Nucl. Phys. B724 (2005) 357

[7] M. Cheng et al. (RBC-Bielefeld), Phys. Rev. D74 (2006) 054507 
[8] C. DeTar, PoS 001 (LAT2008)

R. Gupta [for the HotQCD Collaboration], PoS 170 (LAT2008), arXiv:0810.1764 [hep-lat]. 